inoighbourhood. Messrs. Field and Coi, of Birmingham, have made Hot me' a lens adapted to a most convenient and portable epparatus, which can be fixed with clamp and screw. It has a number of joints of varlous kinds, so arranged that the lens can be focussed at any angle.

In a few hours the circumscribed congestion disappears, leaving small papales and pustules at the point of operation, which may remain visible for some weeks. For this reason, where the hairs are numerous, as upon the upper lip, not more than twelve should be re: moved at a sitting, leaving an interval of a woek or two between ench operation. Where there are but few hairs upon the less prominent regions, all of them may be removed at one sitting; although I may remark that the tediousness of the process soun tells upon the operator.

Minute scars are most apt to occur where it has been found necessary to introduce the needle into the same follicle a number of times, or where hairs situated closely together are removed at one time; but even the most marked cicatrices are scarcely noticeable after the first few. woeks.

Like all operations of a delicate nature, this requires a certain amount of still and mannal dexterity only to be acquired by some experience in its performance ; this, however, is easily gained.

The amount of pain felt differs in different patients, varying accord. ing to the region attacked, or the sensitiveness of the patient; while it is not trivial, it is not unbearable, and a tolerance seems to be established after a few sittings.

In conclusion, I may state that all the cases that have come under my treatment have been most successful. I have recently had an opportunity of examining some patients upon whom I operated six months ago, and find no return of the growth of hair.

\section{A CONTRIBUTION TO THE ETIOLOGY OF DIPHTHERIA.}

Bx G. F. MASTERMAN, Stourport.

IN $1883 \mathrm{I}$ attended a severe case of diphtheria. The patient, who was in her twenty-fifth year, was daily expecting her first confinement, but it was evident, from the nature and gravity of the disease, that her chance of surviving that event, or even living until it could take place, was very small indeed. The diphtheritic growth covered both tonsile, and the pharynx, as far as it could be seen. The accompanying fever was high ; there was extreme depression, and marked albuminuria.

I ascertained, by palpation and auscultation, that the foetus was alive and vigorous, and, also, that the maternal pelvis was large enough to permit speedy delivery, and that the vagina was free from any diphtheritic deposit.

As much milk and egg was given as the patient could take, with sufficient brandy to prevent the tongue from becoming dry, a condition which is, I believe, alike the measure of its necessity and of the quantity to be administered. The fauces were pencilled with a solution of hyposulphite of soda in glycerine; diluted sulphurous acid was used as a gargle, and perchloride of iron given in full doses.

On the fifth day the growth had evidently, from the increased dyspnoes, passed the glottis; and although the general, even more than the local, condition of the patient indicated that tracheotomy could be of little service, I felt it to be my duty to propose that operation to her friends as a last resource, but was rather relieved than chagrined when she and they declined it.

Up to this time there had been no labour-pains; the woman was fast sinking, and, although the foetal heart was still beating strongly, frequent convulsive movements seemed to show that it was feeling the vitiated state of the maternal blood and that its life could only be saved by speedy delivery. I dilated, therefore, the os uteri as rapidly as possible by Barnes's bags, and none too soon, for the woman was already in articulo mortis as I removed the last, After hastily performing tracheotomy to prolong the mother's life for a few minutes and the breathing was temporarily greatly relieved by it), I found that the uterus was so completely relaxed by the approach of death, that my hand passed into its cavity, almost without an effort ; and fortunately at once seizing the feet, I was able to turn, and, with but little force, to bring a fine female child crying into the world. Its unfortunate mother had already expired; a slight, shuddering tremor stirred the flaccid limbs as I divided the funis, but the child had been orphaned before its birth. It was at once removed from the room, and, as soon as possible, sent away from the house.
Now, I am narrating this ease eolely for the sake of its incidence on the pathology of diphtherio, a disease which I had been taught to regard, and had really believed to be, in the words of Tanner, "a specific blood-disease which runs a rapid course," and that the typical growth was a result and an outcome of that morbid condition due to the anteriorly poisoned blood. But here was a child which had existed for above a week on this poisoned blood, filtered, it is true, by the placental dissepiments, but with free endosmotic interchange, yet not only absolutely free from diphtheria, but thoroughly healthy and vigorous, and alive and healthy now.

We know that yariola, syphilis, typhus fever, scarlatina, and other diseases affecting the whole organism are transmitted to the foetus in utero; and I believe I am correct in saying that no woman suffering from any disease of this class can contemporaneously give birth to an uninfected living child. Therefore, if diphtheria bo a blood-disease, this child should surely have shared the fate of its hapless mother.

In 1878, I witnessed an outbreak of diphtheria in a Suffolk village, where the environs were almost incredibly filthy; and in one rocm I saw several children lying scantily covered, and amongst them two little girls whose pudenda were partially coated with the characteristic growth ; and I was struck at the time-although its significance was not so clear to the then as it is now - by the accuracy with which its inner margin was defined by the line of contact when the labia were separated.

I have never seen it on the surface of an ulcer or a blister-denuded cutis, but it has been observed there by others.

In the fauces, it is almost invariably found on the anterior surface of the tonsils, or on the sides of the chink between them and the uvula ; that is, exactly where we should expect to find a growth or exudation due to something from without, and carried thither in the act of inspiration. But, if the disease originate in a morbid bloodchange, and the exudation be a result, then the reverse of this would be anticipated; and-granting that only moist and generally mucous surfaces can be invaded by it-the tongue, the whole interior of the mouth, or the conjunctiva, should preferably disp'ay it.

And again, if the exudation be the result of centrifugal mischief, why should its appearance on an external surface, on one that has been freely blistered or extensively ulcerated, "add greatly to the amount of the disease against which the patient has to contend" (Reynolds' System of Medicine, vol. i, p. 387). The mere exudation of lymph more or less organised can scarcely be of any gravity unless, as in the larynx or bronchi, it is mechanically fatal to life. But if this extension be regarded as the evidence of a fresh or a persistent infection, then its moment is manifestly clear and measurable.

Then as to its source : so many observers have noticed, as I have, an extended area of fungoid growth, sometimes on the wall of the patient's bedroom, or in an adjoining apartment or passage mouldy and damp, or where ammoniacal exhalations from unventilated drains, or heaps of decaying vegetable matter, have supplied the conditions most favourable to fungoid development-that the connection, if not in all primary cases one of cause and effect, is surely more than coincident; not in the sense that the diphtheritic growth is fungoid, for, although fungi are found in it, they seem to be no essential part of it, but that their prolonged inhalation in, perhaps, a depressed state of health, can so specifically influence the surface of the fances or other sensitive areas that a most unhealthy pseudo-foreign growth can be excited thereon, and that this growth is-whatever the ultimate effects on the blood and nerrous system may be-the real origin of the disease. It may seem a strained analogy to compare this growth with that produced on the wild rose by the introduction of the egge- - r some secretion accompanying them-of a Cynips (the briar-burrs, or budegars) for the plant does not seem to be injured by them; but the singular depravity in dovelopment in both cases, and the conversion in each of a smooth, almost polished surface, into one of rugged outgrowth, has often suggested it to me.

And, if these spores be recognised as the exciting cause of the disease, then the way in which it clings to particular houses or rooms, its apparent capriciousness in attacking only one or two out of several persons living in common, and its prevalence during the close damp days of autumn, are accounted for. And, which is of far greater im. portance, by pointing this out to our patients, and insisting that every patch of mouldiness within the house shall be washed with a solution of bichloride of mercury and its cause removed, every damp internal wall dried, and cognate sanitary work sedulously attended to, if they would guard themselves and their children from this terrible disease, we shall, as Dr. Wilks said lately, "in so far as we are successful in so doing, be falfilling one of the highest objects to which anyone can attain." 